\title{
COUNTEREXAMPLE TO A QUESTION ON COMMUTATORS
}

\begin{abstract}
ALAN MCINTOSH
Abstract. We show that it is possible for two selfadjoint operators $A$ and $B$ in a Hilbert space $H$ with bounded commutator $A B-B A$ to have the property that $|A| B-B|A|$ is unbounded (where $|A|$ denotes the positive square root of $A^{2}$ ). The proof reduces to showing that for all natural numbers $n$, there exist a bounded positive operator $U$ and a bounded operator $V$ satisfying $\|U V-V U\| \geqq n\|U V+V U\|$.
\end{abstract}

Introduction. Interest in the above question arises from the fact that if $H=L^{2}(-\infty, \infty), A u=i u^{\prime}$ and $B u=b u$ (where $b$ is an a.e. differentiable function), then $|A| B-B|A|$ is bounded whenever $A B-B A$ is bounded (i.e. whenever $b^{\prime}$ is essentially bounded). Note that $|A| B-B|A|$ is the singular integral operator $(|A| B-B|A|) f(x)$ $=\pi^{-1}$ p.v. $\int(x-y)^{-2}(b(x)-b(y)) f(y) d y$. This is the one-dimensional $L^{2}$ case of a more general theorem of Calderon [1]. It was asked by T. Kato whether this case at least could be proved in an abstract setting, and in particular, whether $|A| B-B|A|$ is bounded whenever $A B-B A$ is bounded.

Although we present two operators with $A B-B A$ bounded and $|A| B-B|A|$ unbounded, the question remains as to whether an abstract proof of Calderon's result can be found. In particular it is clear that in the special case $(A=d / d x, B=b), A B-B A$ commutes with $B$. So it would be interesting to know the answer to the following question: If $A B-B A$ is bounded and commutes with $B$, is $|A| B$ $-B|A|$ necessarily bounded? We comment further on this question at the end of the paper.

Terminology. If $A$ is a linear operator in a Hilbert space $H$, then $D(A)$ denotes the domain of $A$. A linear manifold $X \subset D(A)$ is called a core of $A$ if $X$ is dense in $D(A)$ under the norm $\|u\|_{A}^{2}=\|u\|^{2}+\|A u\|^{2}$. Throughout this paper the scalar field is assumed to be the field of complex numbers $\boldsymbol{C}$.

The result. (I) There exist two linear operators $A$ and $B$ in a Hilbert space $H$ satisf ying:

(i) $A$ is selfadjoint;

Received by the editors October 15, 1970.

AMS 1970 subject classifications. Primary 47B47.

Key words and phrases. Hilbert space, selfadjoint operator, commutator.

Copyright (C) 1971, American Mathematical Society 
(ii) $B$ is bounded and selfadjoint;

(iii) $B(D(A)) \subset D(A)$, and $A B-B A$ is bounded on $D(A)$;

(iv) $|A| B-B|A|$ is unbounded.

[Note. (iii) is equivalent to (iii) $A B-B A$ is defined and bounded on a core of $A$ (provided that (i) and (ii) are satisfied).]

This result can be reduced to the following result on bounded operators:

(II) For all natural numbers $n$ there exist bounded selfadjoint operators $A_{n}$ and $B_{n}$ in a Hilbert space $H_{n}$ satisfying:

(i) $\left\|B_{n}\right\| \leqq 1$;

(ii) $\left\|A_{n} B_{n}-B_{n} A_{n}\right\|=1$;

(iii) $\left\|\left|A_{n}\right| B_{n}-B_{n}\left|A_{n}\right|\right\| \geqq n$.

Indeed (I) is derived from (II) by setting $H=\bigoplus H_{n}, A=\bigoplus A_{n}$ and $B=\bigoplus B_{n}$. (Note that the set $\left\{u=\bigoplus u_{n} \mid\right.$ all but finitely many of the $u_{n}$ are zero $\}$ is a core of $A$.)

Result (II) is a consequence of (III):

(III) For all natural numbers $n$, there exist bounded linear operators $U$ and $V$ in a Hilbert space $K$ satisfying:

(i) $U$ is positive selfadjoint;

(ii) $\|V\| \leqq 1$;

(iii) $\|U V+V U\|=1$;

(iv) $\|U V-V U\| \geqq n$.

To derive (II) from (III), let $H_{n}=K \oplus K$ (with elements of $H_{n}$ denoted by $\left.u_{1} \oplus u_{2}\right)$, let $A_{n}=U \oplus(-U)$, and define $B_{n}$ by $B_{n}\left(u_{1} \oplus u_{2}\right)$ $=V u_{2} \oplus V^{*} u_{1}$ We can represent $A_{n}$ and $B_{n}$ by the matrices

$$
A_{n}=\left[\begin{array}{rr}
U & 0 \\
0 & -U
\end{array}\right] \text { and } B_{n}=\left[\begin{array}{ll}
0 & V \\
V^{*} & 0
\end{array}\right] \text {. }
$$

Then

$$
\begin{aligned}
A_{n} B_{n}-B_{n} A_{n} & =\left[\begin{array}{cc}
0 & U V+V U \\
-\left(U V^{*}+V^{*} U\right) & 0
\end{array}\right], \\
\left|A_{n}\right| & =\left[\begin{array}{cc}
U & 0 \\
0 & U
\end{array}\right] \text { and } \\
\left|A_{n}\right| B_{n}-B_{n}\left|A_{n}\right| & =\left[\begin{array}{cc}
0 & U V-V U \\
U V^{*}-V^{*} U & 0
\end{array}\right] .
\end{aligned}
$$

It is now straightforward to check that (II) is a consequence of (III).

It remains for us to prove (III). Let $k=(3 n+1)^{2}$ and let $m=2^{k}$. Let $K$ be the $m$-dimensional unitary space $C^{m}$. Define $U$ to be the operator in $K$ whose matrix is diagonal with diagonal terms $\lambda_{i}=2^{i}$ $(i=1,2, \cdots, m)$. The operator $V$ is defined to have matrix $\left(v_{i j}\right)$, 
where $v_{i j}=\left(\lambda_{i}+\lambda_{j}\right)^{-1} w_{i j}$, and where the matrix $W=\left(w_{i j}\right)$ is defined inductively by

$$
W_{1}=\left[\begin{array}{cc}
\frac{1}{\sqrt{2}} & -\frac{1}{\sqrt{2}} \\
0 & 0
\end{array}\right], \quad W_{r+1}=\left[\begin{array}{cc}
W_{r}+Y_{r} & -Y_{r} \\
0 & W_{r}
\end{array}\right],
$$

and $W=W_{k}$. The matrix $Y_{r}$ is the $2^{r} \times 2^{r}$ matrix with terms in the bottom row equal to $2^{-(r+1) / 2}$, and all other terms zero. So, for example,

$$
W_{2}=\left[\begin{array}{rrrr}
\frac{1}{\sqrt{2}} & -\frac{1}{\sqrt{2}} & 0 & 0 \\
\frac{1}{2} & \frac{1}{2} & -\frac{1}{2} & -\frac{1}{2} \\
0 & 0 & \frac{1}{\sqrt{2}} & -\frac{1}{\sqrt{2}} \\
0 & 0 & 0 & 0
\end{array}\right]
$$

The matrix $W$ is constructed so that (i) the nonzero rows, considered as vectors in $K$, form an orthonormal set, (ii) if $W_{a}$ is the matrix $\left(\left|w_{i j}\right|\right)$, then $\left\|W_{a}(1,1,1, \cdots, 1)\right\|=\sqrt{ }(k m)=\sqrt{ } k\|(1,1,1, \cdots, 1)\|$, and (iii) $w_{i j} \geqq 0$ if $i \geqq j$, and $\leqq 0$ if $i<j$.

We now check the four properties listed in (III). The first is clear.

$$
\begin{aligned}
\|V\|^{2} & \leqq \sum_{i} \sum_{j} v_{i j}^{2}=\sum_{i} \sum_{j}\left(\lambda_{i}+\lambda_{j}\right)^{-2} w_{i j}^{2} \\
& \leqq \sum_{i} \lambda_{i}^{-2} \sum_{j} w_{i j}^{2}<1 .
\end{aligned}
$$

(iii) The fact that the nonzero rows of $W$ form an orthonormal set implies that $\|W\|=1$. Now $W=U V+V U$, so $\|U V+V U\|=1$.

(iv) Denote $U V-V U$ by $S=\left(s_{i j}\right)$. Then

$$
s_{i j}=\left(\lambda_{i}-\lambda_{j}\right) v_{i j}=\left(\lambda_{i}-\lambda_{j}\right)\left(\lambda_{i}+\lambda_{j}\right)^{-1} w_{i j} \geqq \frac{1}{3}\left|w_{i j}\right| \quad \text { if } i \neq j
$$

(because $\left(\lambda_{i}-\lambda_{j}\right)\left(\lambda_{i}+\lambda_{j}\right)^{-1} \geqq \frac{1}{3}$ if $i>j$, and $\leqq-\frac{1}{3}$ if $i<j$ ).

$$
\begin{aligned}
\therefore s_{i j}+\frac{1}{3} \delta_{i j} & \geqq \frac{1}{3}\left|w_{i j}\right|, \quad 1 \leqq i, j \leqq m . \\
\therefore\left\|\left(S+\frac{1}{3} I\right)(1,1, \cdots, 1)\right\| & \geqq \frac{1}{3}\left\|W_{a}(1,1, \cdots, 1)\right\|=\frac{1}{3} \sqrt{ }(k m) . \\
\therefore\|S(1,1, \cdots, 1)\| & \geqq \frac{1}{3}(\sqrt{ } k-1) \sqrt{ } m . \\
\therefore\|S\| & \geqq n \quad\left(\because k=(3 n+1)^{2}\right) .
\end{aligned}
$$

This completes the proof. 
Remark. It was asked in the introduction whether the situation is altered by adding the extra condition: $B$ commutes with $A B-B A$.

It is interesting to note that a counterexample cannot be constructed along the above lines in this case. For if $A$ and $B$ are bounded selfadjoint operators such that $B$ commutes with $A B-B A$, then $A B-B A=0$ (see $[2$, p. 4]).

ADDED IN PROOF. 1. We may require that the $V$ defined in (III) be selfadjoint (for if $V$ satisfies the equations in (III), then either the real or imaginary part of $V$ satisfies the same equations with $n$ replaced by $\left.\frac{1}{2} n\right)$. Hence there exist selfadjoint operators $U$ and $V$ in $C^{m}$ such that $U$ is positive and $\|U V-V U\| /\|U V+V U\|$ $\geqq$ const $(\log m)^{1 / 2}$. The author has learned by private communication that W. Kahan has obtained such operators satisfying $\|U V-V U\| /\|U V+V U\| \geqq \pi^{-1} \log m$. Kahan has also shown that if $Z$ is an $m \times m$ matrix with real eigenvalues, then $\left\|Z-Z^{*}\right\| /\left\|Z+Z^{*}\right\|$ $\leqq 0.1+\log _{2} m$.

2. The author has applied (III) to prove the existence of a regularly accretive operator $A$ such that $A^{1 / 2}$ and $A^{*(1 / 2)}$ have different domains. Details will be published elsewhere.

\section{REFERENCES}

1. A. P. Calderon, Commutators of singular integral operators, Proc. Nat. Acad. Sci. U.S.A. 53 (1965), 1092-1099. MR 31 \#1575.

2. C. R. Putnam, Commutation properties of Hilbert space operators and related topics, Springer-Verlag, New York, 1967. MR 36 \#707.

Macquarie University, North Ryde, N.S.W., 2113, Australia 